\title{
Fuzzy multivariate forecasting of the implementation of regional development programs for the agro-industrial complex
}

\author{
Vadim Lomazov ${ }^{1,2 *}$, Olga Ivashchuk ${ }^{2}$, Alexander Lomazov² and Olga Akupiyan ${ }^{1}$ \\ ${ }^{1}$ Belgorod State Agricultural University, 308503, Vavilova st, 1, Mayskiy, Belgorod region, Russia \\ ${ }^{2}$ Belgorod National Research University, 308015, st. Pobedy, 85, Belgorod, Russia
}

\begin{abstract}
The article is devoted to the problems of improving digital intellectual tools for managing the implementation of socio-economic and technological programs aimed at developing the agro-industrial cluster of the regional economy. The aim of the work is to develop a procedure for forecasting the implementation of programs based on the data of the previous stages and knowledge, reflecting the specifics of agricultural production. To describe the indicators of the current and projected state of the regional agro-industrial complex, it is proposed to use the apparatus of the theory of linguistic variables, which makes it possible to use expert technologies for filling the knowledge base and allows us to take into account the high level of uncertainty characteristic of the agricultural market. The links between current and projected performance are represented by fuzzy production rules. The fuzzy inference procedure used in forecasting (based on the Mamdani algorithm) is built in the form of an interpreted fuzzy multilayer neural network. The preliminary results of using the developed procedure as part of a research prototype of an information-analytical system may indicate its effectiveness. The practical significance of the developed toolkit is due to the possibility of its use as a means of intellectual support for making scientifically grounded management decisions on the implementation (taking into account possible adjustments) of development programs for the regional agro-industrial complex.
\end{abstract}

\section{Introduction}

Currently, an important instrument of state regulation and planning is the implementation of programs for sustainable socio-economic and technological development of the country and regions [1]. Regional programs for sustainable development of the agricultural sector of the economy are focused on comprehensive support of modern trends in agricultural production, including the biologization of agriculture (production of environmentally friendly products [2], soil regeneration [3], reduction of agrogenic pollution [4], use of renewable energy sources [5,6]). However, the use of new agricultural production technologies is associated with a number of problems (for example, often, insufficient

\footnotetext{
* Corresponding author: vlomazov@yandex.ru
} 
scientific validity, low economic efficiency, technical and technological unavailability, qualified personnel deficiency, practical experience deficiency, etc.), which requires constant monitoring, forecasting and (if necessary) corrections of the program progress ([7]). To manage the implementation of regional programs for the development of the agroindustrial complex, effective digital intellectual (knowledge-oriented) information and analytical support is needed, which is currently underdeveloped.

The purpose of this study is to develop a procedure for forecasting the implementation of programs for the sustainable development of the regional agro-industrial complex on the basis of current monitoring data and knowledge reflecting the specifics of agricultural production. A multivariate (taking into account the influence of external factors) forecast of the results (and risks) of the introduction of the latest agricultural technologies in the region makes it possible to make timely management decisions that increase efficiency and reduce the possible consequences of negative factors. The proposed use of models and methods of fuzzy linguistic analysis is due to the high level of uncertainty characteristic of forecasting tasks in the agricultural sector.

\section{Materials and Methods}

\subsection{Statement of the multivariate forecasting problem}

Let us consider the task of making a medium-term forecast of the consequences of the implementation of the regional program for the development of the agro-industrial complex, which requires taking into account the factors of the internal environment and the factors of the external micro-and macro-environment and is (by the number of factors taken into account) the most difficult of the forecasting tasks (Fig.1).

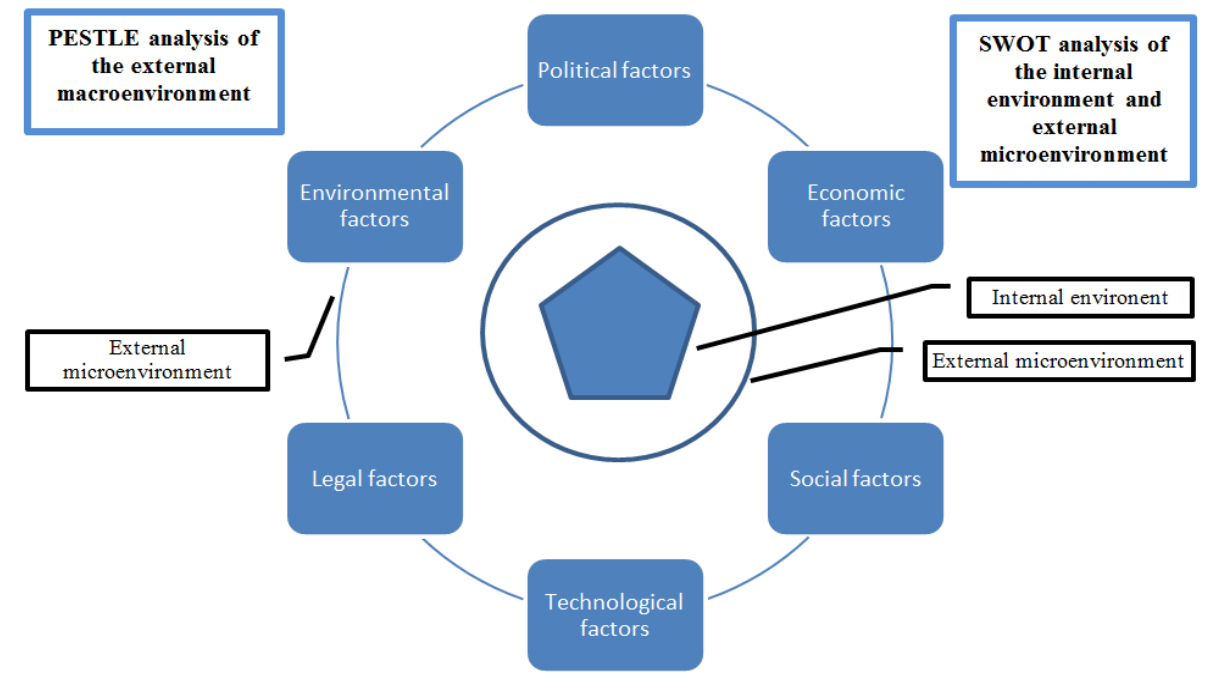

Fig. 1. Accounting for internal and external factors of the regional agricultural economy using SWOT and PESTLE analysis tools

Let the PESTLE and SWOT analysis [7] of the regional agro-industrial complex determine:

- complex of main characteristics - Char $=<C h_{1}, C h_{2}, \ldots, C h_{n}>$;

- complex of internal environment factors - FactIntMed $=<$ Fim $_{1}$, Fim $_{2}, \ldots$, Fim $_{m}>$; 
- complex of external environment factors - FactExtMed $=<$ Fem $_{1}, \mathrm{Fem}_{2}, \ldots, \mathrm{Fem}_{p}$ $>$.

Along with the set of target indicators $\operatorname{TargInd}=<\operatorname{Targ}_{1}, \operatorname{Targ}_{2}, \ldots, \operatorname{Targ}_{s}>$, which defines the program under consideration, the characteristics Char, FactIntMed, FactExtMed make up the set of input variables of the procedure for making a medium-term forecast:

$$
\text { InVar }=<\text { Char, FactIntMed, FactExtMed, TargInd }>
$$

The output variables of the procedure are the predicted values of the characteristics of the regional agro-industrial complex OutVar $=$ CharProgn $=\left\langle C h p_{1}, C h p_{2}, \ldots, C h p_{r}\right\rangle$.

Fixing the existing initial state of the regional agricultural economy Char*, the target indicators of the TargInd* program and the internal factors of the development of FactIntMed*, we will associate the uncertainty only with the influence of external factors, each set of which FactExtMed* will correspond to some variant of the forecast. Although the use of the proposed formalized forecasting procedure makes it possible to consider a large number of options, however, in many cases it is advisable to limit the traditional study of the optimistic, basic (most likely) and pessimistic option, choosing the corresponding sets of values of external factors. Further we will consider the problem of forecasting for a specific option, bearing in mind that the analysis of other scenarios is performed by substituting the corresponding sets of values of external factors for the input of the procedure.

\subsection{Linguistic representation and fuzzy knowledge production model}

We will assume that the characteristics, indicators, and factors considered as linguistic variables allow for quantitative measurement (in points from 1 to 10) and verbal measurement by the values of the term set $\mathrm{T}=\{$ low, medium, high $\}$, reflecting the level (degree of influence) of each characteristic (factor).

The relationship between verbal and quantitative variables (semantic rule) is given by the Gaussian correspondence function (the membership function of a fuzzy subset of the underlying numerical set $\mathrm{U}=[1,10]$ )

$$
\left.\mu(x)=\exp \left(-\left(x-x^{0}\right) / \sigma\right)^{2}\right)
$$

where $x^{0}$ is the center and $\sigma$ is the width of the Gaussian curve (the values may vary for different linguistic variables).

Knowledge about the program and regional agroeconomics is presented as a database of fuzzy production rules, which (in accordance with [8]), in the absence of adaptive program management, look like:

$$
\begin{gathered}
\left.\left.R^{j}, \eta^{t}: \quad \text { IF (Char, FactIntMed, FactExtMed, TargInd }\right)-X^{j} \in T^{(n+m+p+s)}\right) \\
\text { THEN (CharProgn } \left.-Y^{j} \in T\right), \\
j=1,2, \ldots, N,
\end{gathered}
$$

where $X=\left(x_{1}, x_{2}, \ldots, x_{n+m+s+p}\right), Y=\left(y_{1}, y_{2}, \ldots, y_{r}\right)$ are vectors of verbal (from the set T) values that determine the initial state, effective internal and external factors, target indicators and the predicted state of the regional agroeconomy, and $\eta_{j j}$ is the degree of confidence of the rule $\left(0 \leq \eta_{j \mathrm{j}} \leq 1\right)$.

Another way to represent knowledge about the subject area is to use the apparatus of fuzzy relations. In this case (using the Larsen implication model), the fuzzy forecast model will have the form:

$$
\mu_{\text {InVar } \rightarrow \text { OutVar }}=\left(\mu_{\text {InVar }}\right)\left(\mu_{\text {OutVar }}\right)
$$


where $\mu_{\text {InVar }}, \mu_{\text {OutVar }}$, are the term-matching functions from the term-set $\mathrm{T}$ for the input and output variables, and $\mu_{\text {InVar } \rightarrow \text { OutVar }}$ is the term-matching function for the implication relation connecting the input and output variables:

$$
0 \leq \mu_{\text {InVar }}, \mu_{\text {OutVar }}, \mu_{\text {InVar } \rightarrow \text { OutVar }} \leq 1
$$

Fuzzification means the transition from the numerical values of the input variables InVar* obtained as a result of SWOT and PESTLE analysis to terms (verbal values) with matching values found when substituting numerical values into the Gaussian function $\mu_{\text {InVar }}\left(X^{*}\right)$.

Fuzzy inference (implication) is the determination of the value of the term matching function for the output variables $\mu_{\text {OutVar* }}(y)$ by the formula

$$
\mu_{\text {OutVar }}(y)=\mu_{\text {OutVar }}(y) \times \mu_{\text {OutVar }}\left(x^{*}{ }_{1}\right) \times \mu_{\text {OutVar }}\left(x^{*}{ }_{2}\right) \times \ldots \times \mu_{\text {OutVar }}\left(x^{*}{ }_{n+m+p+s}\right)
$$

Finding the numerical values of the output variables (defuzzification) in accordance with the middle center method is performed using the formula

$$
\text { OutVar }^{*}=\left(\text { OutVar }^{* 1} P^{1}+\text { OutVar }^{* 2} P^{2}+\ldots+\text { OutVar }^{* N} P^{N}\right) /\left(P^{1}+P^{2}+\ldots+P^{N}\right)
$$

where

$$
\left.\left.P^{j}=\exp \left(-\left(x^{*}{ }_{1}-x^{* j}{ }_{1}\right)^{2} / \sigma^{j}{ }_{1}\right)^{2}\right) \times \ldots \times \exp \left(-\left(x^{*}{ }_{n+m+p+s}-x^{* j}{ }_{n+m+p+s}\right)^{2} / \sigma^{j}{ }_{n+m+p+s}\right)^{2}\right)
$$

\section{Results and Discussion}

Operations of fuzzification, fuzzy implication, and defuzzification can be conveniently implemented as an interpreted fuzzy multilayer neural network, the architecture of which is shown in Fig. 2.

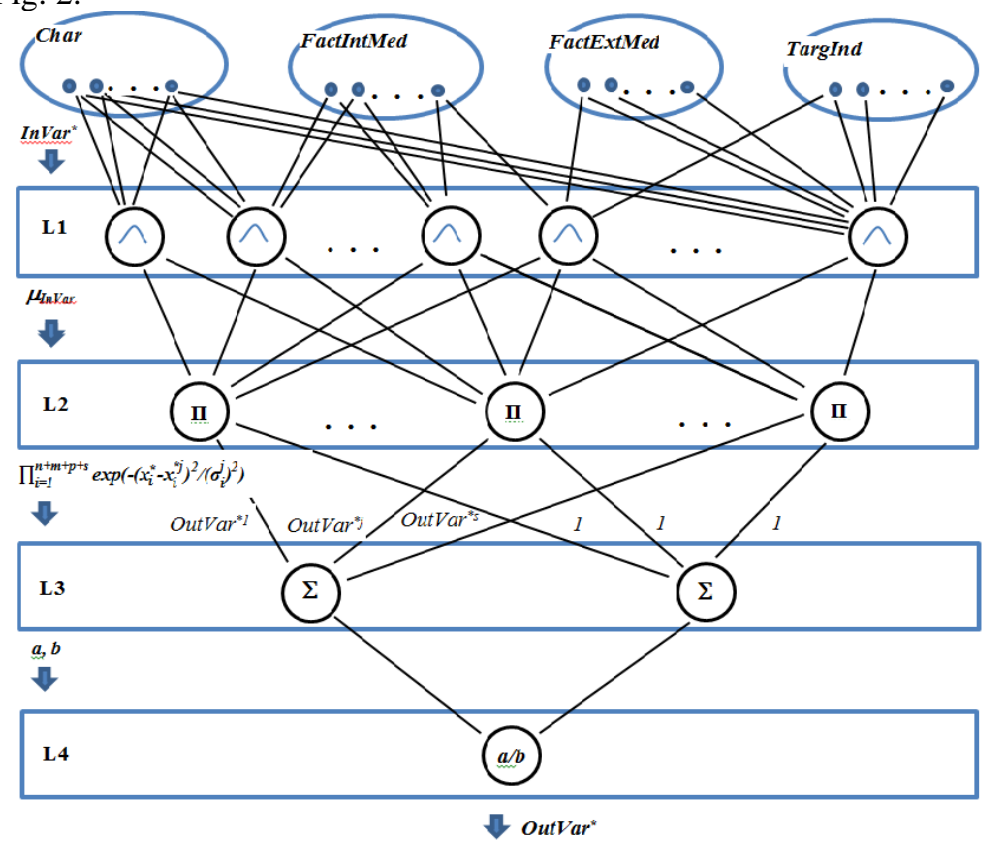

Fig. 2. A scheme for the implementation of fuzzy neural network forecasting of the results of the implementation (within the framework of a specific version) of the regional program for the development of the agro-industrial complex 
The input of the elements of the first layer (L1) is supplied with the numeric values of the input variables, which are used to determine the values of $\mathrm{InV} \operatorname{InVar}$ passed to the elements of the second layer (L2). The L2 link configuration corresponds to the rule base, and the multipliers correspond to fuzzy implication operations. Defuzzification is represented by the elements of the third (L3) and fourth (L4) layers, and the outVar*j centers play the role of connection weights from the L2 elements to the L3 elements. L3 implements summation, and L4 implements division, the result of which is the numeric value of the output variable outVar*. It should be noted that outVar* is a vector quantity, each component of which represents one of the predicted (within the framework of the considered scenario) characteristics, and in fact it is necessary to perform the procedure shown in Figure 2 for each component. The advantage of the neural network implementation of the prediction problem solution is the possibility of self-learning the network on the elements of the training sample. Since the considered neuro-fuzzy network is a network of direct signal propagation, the method of backward error propagation can be used for its training [9].

Preliminary results of using the developed procedure as part of research prototype of the information and analytical system may show its effectiveness. The practical significance of the developed tools is due to the possibility of its application as an instrument of intellectual support for making scientifically based management decisions on the implementation (taking into account possible adjustments) of development programs for the regional agroindustrial complex.

In the framework of further research, the relative values obtained in dimensionless form (in points) of the predicted characteristics can be used for further analysis by comparing them with the initial (at the beginning of the program) and the desired relative values of the corresponding characteristics when solving problems of modeling and synthesis of regional agricultural systems $[10,11]$.

The reported study was funded by RFBR, project number 20-07-00855.

\section{References}

1. J. Blewitt, Understanding Sustainable Development (Routledge, London, 2017)

2. R. Blair, Organic Production and Food Quality: A Down to Earth Analysis (Wiley-Blackwell, Oxford, 2012)

3. R. Lal, B.A. Stewart (Eds.), Principles of Sustainable Soil Management in Agroecosystems (CRC Press, 2013)

4. A. Abbasi, A. Sajid, N. Haq, S. Rahman, Z.T. Misbah, G. Sanober, M. Ashraf, A.G. Kazi, Improvement of Crops in the Era of Climatic Changes, 1: 347-387, (2014)

5. A. Wellinger, J. Murphy, D. Baxter The biogas handbook: science, production and

6. Applications (Woodhead Publishing, Cambidge, 2013)

7. V.A. Lomazov, V.I. Lomazova, I.V. Miroshnichenko, D. A. Petrosov, A.L. Mironov, IOP Conf. Ser.: Earth Environ. Sci., 659, 012111 (2021)

8. M. Kuhn, K. Johnson Applied Predictive Modelling (Springer International Publishing, 2013)

9. Ch. Osita, I. Onyebuchi, N. Justina, International Journal of Innovative and Applied Research, 2(9): 23-32 (2014)

10. C. Grosan, A. Abraham, Intelligent Systems: A Modern Approach (Springer-Verlag Berlin Heidelberg, 2011)

11. Halsey Wilson, Artificial intelligence (Grey House Publishing, 2018)

12. D.A. Petrosov, V.A. Lomazov, A.I. Dobrunova, S.L. Matorin, V.I. Lomazova, Biosciences Biotechnology Research Asia, 12(3): 2971-2981 (2015)

13. V.A. Lomazov, D.A. Petrosov, A.I. Dobrunova, V.I. Lomazova, S.I. Matorin, International Journal of Applied Engineering Research, 11(3): 1867-1873 (2016) 